\title{
Formation of AGS in an Airlift Reactor with Divided Funnels Under Combined Selective Pressure
}

\author{
Nik Azimatolakma Awang 1,2,", Md. Ghazaly Shaaban ${ }^{2}$, Lee Choon Weng ${ }^{3}$, \\ Bong Chui Wei ${ }^{3}$
}

${ }^{1}$ School of Civil Engineering, Engineering Campus,

Universiti Sains Malaysia, 14300 Pulau Pinang, MALAYSIA

${ }^{2}$ Department of Civil Engineering, Faculty of Engineering,

University of Malaya, 50603 Kuala Lumpur, MALAYSIA

${ }^{3}$ Institute of Biological Sciences, Faculty of Science,

University of Malaya, 50603 Kuala Lumpur, MALAYSIA

*Corresponding Author

DOI: https://doi.org/10.30880/ijie.2019.11.01.011

Received 15 May 2018; Accepted 18 July 2018; Available online 10 May 2019

\begin{abstract}
Understanding on the competence of aerobic granules sludge (AGS) system to recover the granulation process when instability occurs remained scarce since current research works on continuous variation in the organic loading rate (OLR) applied have been rarely reported. Thus, this study determines the dynamic granulation process and stability of AGS system by combining selective pressure generated by variation of OLR from 0.26 to $0.81 \mathrm{~kg} \mathrm{CODs} \mathrm{m}^{-3} \mathrm{~d}^{-1}$, use of an airlift reactor with divided funnel having a low height/diameter (H/D) ratio of 4.4 and low hydrodynamic shear force (superficial air velocity, SAV of $0.7 \mathrm{~cm} \mathrm{~s}^{-1}$ ). Matured AGS was attained after 67 days of the reactor setup with an optimal sludge volume index (SVI) of $33 \mathrm{ml} \mathrm{g}^{-1}$ and 15 min of settling time. The AGS system was generally stable between day 41 to 67 at average mixed liquor suspended solids (MLVSS) of $0.85 \mathrm{~g} \mathrm{~L}^{-1}$ and sludge retention time of 6 days. The next generation sequencing (NGS) analysis indicated that the shift in microbial community performing denitrification process from Rhodocyclaceae to Comamonadaceae class was accommodated with changes in aerobic granules size from $147 \mu \mathrm{m}$ to over $336 \mu \mathrm{m}$.
\end{abstract}

Keywords: Aerobic granular sludge, airlift reactor, microbial structure, next generation sequencing, sewage

\section{Introduction}

Aerobic granular sludge (AGS) can be defined as a self-immobilization of bacteria to aggregate with suspended biomass to form discrete well defined granules without the use of carrier material [1]-[3]. Compared to anaerobic granular sludge which has been widely applied, the instability of aerobic granules in long term operational bioreactors [1] had become the bottleneck for its practical application [4]. The stability of aerobic granules is defined as the ability of microbial granules to resist hydrodynamic and mechanical shear force [5]. The state of aerobic granules stability can be measured in terms of bioactivity, microbial community, and extracellular polysaccharides (EPS) composition and distribution, and granular characteristic [6]. Table S1 presents a summary of current research works regarding the operating conditions strategized to enhance the stability of aerobic granules system.

According to Tao et al. [7], the formation mechanism of aerobic granules can be categorized into three phases, namely lag, granulation and maturation. in which the aerobic granules reach the largest size and continually constant during the maturation period. In contrary, the model for aerobic granules formation developed by Verawaty et al. [8] showed that matured aerobic granules will subsequently disintegrate after reaching a certain critical size and causing an 
overall reduction in granular sizes. A model developed by Verawaty et al. [8] was further approved by Awang and Shaaban [9] which exhibited granulation, maturation and disintegration as a cyclic process. The difference in the mechanism proposed might be attributed by different type of substrate used, either synthetic wastewater with constant organic loading rate (OLR) [7] or real wastewater with continuous variation in OLR [8,9]. Each type of substrate has a maximum OLR that can be treated in the bioreactor system without affecting the aerobic granules stability. However, the understanding on the capacity of the system to recover the granulation process when instability occurs remained scarce since current research works on continuous variation in the OLR applied have been rarely reported [10]. Most of the works regarding stability as presented in Table S1 have been performed using synthetic wastewater with constant OLR except for Corsino et al. [11] and Liu and Tay [12] which used brewery and industrial wastewater, respectively.

Although Pronk et al. [13] have successfully implemented aerobic granules technology for the treatment of sewage at full-scale with detailed description of the process, conversions, energy usage and design consideration, relatively, but lack of information was reported regarding the characteristics of AGS with respect to OLR variations. A review by Nancharaiah and Reddy [14] suggested that the future research on aerobic granules should be directed towards discovering formation mechanisms, improving formation of aerobic granules in low-strength sewage and wide-spread implementation of aerobic granules systems in sewage and industrial wastewater treatment.

In this context, the objective of the present study is to determine the dynamic granulation process and stability of aerobic granules system when developed under combined selective pressure generated by variation of OLR, reactor with a low height/diameter (H/D) ratio and low hydrodynamic shear force. The relationship of extracellular polymeric substances (EPS) distribution and microbial community structure at different phases of aerobic granulation process were also evaluated.

\section{Material and Methods}

\subsection{Reactor Setup and Operation}

It was reported that reactor with H/D ratio of over 10 was mostly used in the literature [15] in order to meet the requirement of the minimal SAV of $1.2 \mathrm{~cm} / \mathrm{s}$ for a successful formation of AGS [16]. Therefore, this study has defined that a reactor with an H/D below than 5 was considered to be low. An airlift reactor with divided funnels as showed in Fig. S2(a) was proposed for the optimization of aerobic granules formation in reactor with low H/D ratio of 4.4 and run under low SAV. The internal diameter of the airlift reactor was $11 \mathrm{~cm}$ and the diameter of the divided funnels was 8.5 $\mathrm{cm}$. The divided funnels comprised two sections with the same length $(10 \mathrm{~cm})$ and effluent discharging point located between the gaps of the divided funnels. In order to alleviate the problem with friction of aerobic granules in downstream area, $2.5 \mathrm{~cm}$ gap was set between the divided external funnels and sidewall of the reactor.

The operating strategies comprised two phases. In Phase 1, the cycle time was 4 hours with filling, reaction, settling and discharge time were fixed to $30 \mathrm{~min}, 185 \mathrm{~min}, 15 \mathrm{~min}$ and $6 \mathrm{~min}$, respectively. During Phase 2 , the cycle time was changed to 3 hours with filling (30-10 min) and reaction times (125-145 min) changed accordingly to the performance of aerobic granules. Aeration was achieved by injecting air from the bottom of the reactor through a finebubble porous stone. The low air velocity resulted in a low SAV of $0.7 \mathrm{~cm} \mathrm{~s}^{-1}$ during reaction time. After settling time, $2.25 \mathrm{~L}$ of the supernatant was discharged which resulting in a volumetric exchange ratio of $50 \%$. The experiment was operated under room temperature without $\mathrm{pH}$ control. A certain volume of sludge (around $50 \mathrm{~mL}$ ) in the reactor was periodically taken just before the aeration time ended and stored in $-20^{\circ} \mathrm{C}$ for further investigation.

The given flow patterns for bubble column (Fig. S2(b) to Fig. S2(c)) were based on a report by Liu and Tay [17], [18] which described liquid upflow pattern as a homogenous circular flow along the reactor height, in which longer flowing trajectories in SBR with higher H/D ratio will provide better effective hydraulic attrition for microbial aggregation to form aerobic granules. The flow pattern for airlift reactor with divided funnels used in this study (Fig. S2(d)) was derived based on Liu and Tay [17] and Zhou et al. [19] where liquid turns flow direction when it reaches an inclined part of the funnels, resulting in two circular flowing trajectory inside the reactor. Under combined selective pressure of low SAV and low H/D ratio, the airlift reactor with divided funnels can deliver a higher shear force and better mixing compared to bubble column type reactor.

\subsection{Sewage and Seed Preparation}

Sewage and activated sludge were collected from a sewage treatment plant operated by Indah Water Konsortium Sdn. Bhd (Malaysia), located at Damansara and Pantai 1, respectively. The raw sewage and activated sludge were taken directly from primary clarifier and aerobic tank, respectively. The characteristics of the sewage are as follows: total chemical oxygen demand (COD) of 470-163 $\mathrm{mg} \mathrm{L}^{-1}$, soluble chemical oxygen demand (CODs) of 231-86 mg L $\mathrm{L}^{-1}$, 5day biochemical oxygen demand $\left(\mathrm{BOD}_{5}\right)$ of $54-140 \mathrm{mg} \mathrm{L}^{-1}$, suspended solids (SS) of 32-136 mg L ${ }^{-1}$ and ammonium $\left(\mathrm{NH}_{4}{ }^{+}\right)$of $22-53 \mathrm{mg} \mathrm{L}^{-1}$. At initial stage, $2.3 \mathrm{~L}$ of activated sludge after aeration for 1 day was used as seed, resulting in an initial concentrated mixed liquor suspended solids (MLSS) of $1.7 \mathrm{~g} \mathrm{~L}^{-1}$. 


\subsection{Analytical Methods}

Parameters such as $\mathrm{BOD}_{5}$, CODs, MLSS, mixed liquor volatile suspended solids (MLVSS), SS and sludge volume index (SVI) were analysed according to APHA standard methods [20]. In this study, CODs was used instead of total COD due to a huge increment for total COD compared to CODs when the SS value increased. A high SS value contributes to an increase in the COD value, since more organic and inorganic materials are oxidized by potassium dichromate [9]. Ammonium, nitrite and nitrate were determined by the photometric determination using Merck test kit. The morphology of aerobic granules was observed using field emission scanning electron microscope (FESEM), where sample preparation was described by Awang and Shaaban [15]. Aerobic granules sizes were measured under a digital microscope, DSX500 Opto with 5x magnification lenses. The size, geometric shapes and angles on screen were measured and analysed by an image acquisition of 2D (OLYMPUS Stream image analysis software).

Multiple staining schemes for aerobic granules were employed to determine the distribution of EPS. General principles in designing a multicolour staining scheme for granules have been presented by Chen et al. [21] with special precautions taken to avoid overlapping of excitation and emission wavelengths of fluorophores (dyes). In this study, SYTO 63 (Product ID, S11345) and FITC (Product ID, F6434) were purchased from Life Technologies. Calcofluor white (Product ID, 18909) was purchased from Sigma. The stained aerobic granules were kept in cold room at temperature of $5{ }^{\circ} \mathrm{C}$. The whole aerobic granules images were visualized under confocal laser scanning microscope (CLSM; Leica Tcs Sp5 li model) with corresponding excitations and emissions. Z-sectioning was performed on whole granules and rendered three-dimensionally by using Las Af Lite software.

\subsection{Microbial Community Identification}

The shift in the microbial community was analysed using polymerase chain reaction (PCR) coupled with nextgeneration sequencing (NGS) method. NGS is a new method that helps to identify microbial community based on percentage quantity rather than percentage similarity. Aerobic granules were centrifuged at room temperature for $30 \mathrm{sec}$ at $10,000 \mathrm{~g}$. Water was removed to provide approximately $0.25 \mathrm{~g}$ of biomass. DNA was isolated from aerobic granules using PowerSoil ${ }^{\circledR} \mathrm{DNA}$ isolation kit and kept in a freezer at a temperature of $-20^{\circ} \mathrm{C}$ until microbial identification was carried out.

The platform for NGS used in this study was based on Illumina Miseq. Methods described for DNA quality control, bioinformatics analysis and taxonomic classification were provided by Sango Biotech (Shanghai) Co., Ltd. The DNA concentration and purity were measured using agarose gel electrophoresis technique. DNA concentration required for PCR reaction was quantified using Qubit 2.0 DNA kit. The primer for PCR was designed using a blend of universal primer of Illumina Miseq sequencing platform. Agarose gel electrophoresis was run to test the PCR products, and DNA was recovered by Sangon agarose recover kit. Once again, Qubit 2.0 was used to quantify the recovered products based on a mixture of 1:1 ratio with DNA concentration. The mixed sample was shaken and later used as subsequent sample for library construction and sequencing.

PRINSEQ software was used to truncate low-quality data in order to improve merge ratio for subsequent sequences. Flash software was used to fuse dual-terminal sequences and split the data to samples by barcodes. Primer and barcodes were then trimmed from the resulting sequences using PRINSEQ software. UCHIME software and sequences in Silva data as template were used to filter out PCR chimeras. Only samples with sequences length of over $450 \mathrm{bp}$ were used for taxonomic classification analysis. The sequences readings were clustered into operational taxonomic units (OTU) based on the distance between sequences. OTU clustering software used was UCLUST. Sequence similarity was set at 0.97 , and OTU was considered to be as close as possible to genus. The representative sequences from each OTU were subjected to RDP Classifier software, and Greengenes database was used to classify taxonomic assignment based on Bergey's taxonomy.

\section{Results and Discussion}

\subsection{Size and Morphology Development}

Fig. 1(a) exhibits the deviation in aerobic granules size at different days which was reflected in the length of the box plot. On day 0 , the inoculate seed was activated sludge with median size of $154 \mu \mathrm{m}$. Dissipation of activated sludge occurred within 24 hour of start-up and resulted in pin flocs formation on day 1. The aggregation of pin flocs started on day 1 , and by day 8 the pin flocs of median size started to increase from 24 to $99 \mu \mathrm{m}$. Fluffy aerobic granules started to appear after 32 days, but with apparent existence of filamentous microorganism protruding from the aggregates. The deviation in aerobic granules size on day 50 between 264 to $459 \mu \mathrm{m}$ was within the range of day 32 (151 to $545 \mu \mathrm{m}$ ). This suggested that the densify process of fluffy aerobic granules has started. This was in line with pattern of sludge volume index (SVI) profile (Fig. 1(b)) that exhibited small deviations between 5 min intervals of settling time after 22 days of the formation process. Moreover, the morphology of aerobic granules on day 50 (Fig. 2(b)) exhibited a cauliflower-like structure. Meanwhile, Fig. 2(a) displays aerobic granules on day 32 which have a long straggler shape indicating an aggregation of small aerobic granules along the filamentous microorganism as backbone. Similar 
cauliflower-like structure was also observed by Liu et al. [22] when aerobic granules were developed at substrate with $\mathrm{COD} / \mathrm{N}$ ratio less than 5 .
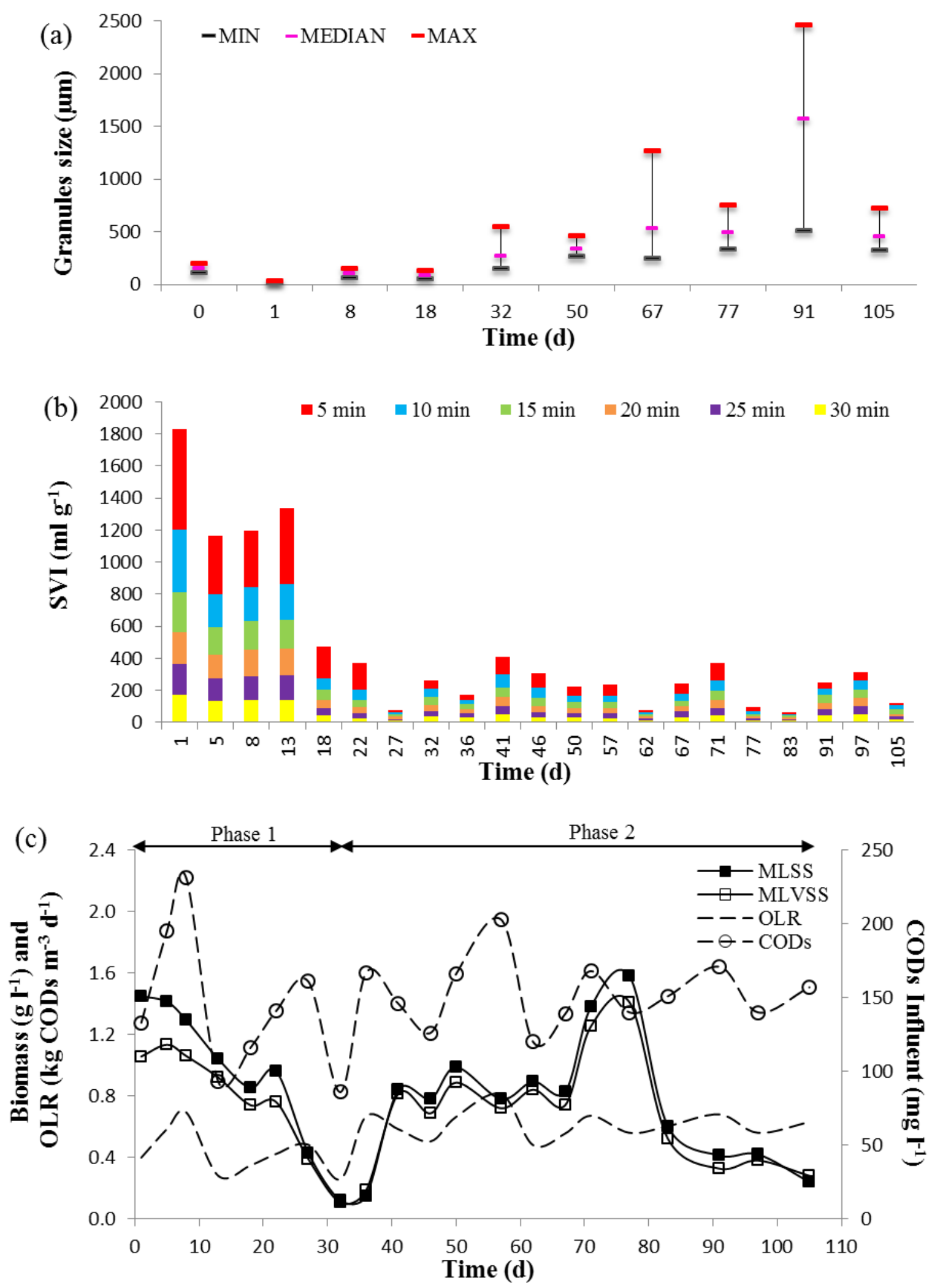

Fig. 1 - (a) Deviation in aerobic granules size (b) Profile of SVI at every 5 min intervals of settling time (c) Profile of biomass (MLSS and MLVSS), OLR and CODs influent 
Aerobic granules size continued to increase up to $1237 \mu \mathrm{m}$ on day 67 , with a large number of small aerobic granules of less than $532 \mu \mathrm{m}$ were predominantly present in the reactor. This might be attributed to the densify process which contributed to a clear outline boundary of aerobic granules. Thus, it can be concluded that mature aerobic granules were achieved on day 67. According to Verawaty et al. [8], mature aerobic granules will subsequently disintegrate after reaching a certain critical size and thus causing an overall reduction in granular sludge. This indeed occurred on day 77 in which the size of mature aerobic granules on day 67 decreased and varied between 333 and 750 $\mu \mathrm{m}$. The morphology of aerobic granules on day 77 (Fig. 2(c)) was more likely exhibited the detachment of biomass from parents aerobic granules, and this further confirmed the disintegration of mature aerobic granules on day 67 . A close-up image on aerobic granules morphology at day 77 demonstrated the appearance of peritrichous ciliates surrounded by various degrees of rod and cocci shape bacteria. According to Weber et al. [23], ciliates stalks serve as backbone in the granule-forming process since bacteria use them as a substratum to grow. At day 91, the aerobic granules continued to increase in size, with diameter varying between 511 and $2463 \mu \mathrm{m}$. This signified the aggregation between small and large granules via bridging, which in turn led to irregular shape and diffused granular structure. Mature aerobic granules again were predominant in the reactor on day 105 with diameter varying between 326 and 728 $\mu \mathrm{m}$. Based on microscope and FESEM images, the mature aerobic granules produced on day 105 exhibited a tiny oval or rod shape form instead of the desired sphere shape.

SVI profile in Fig. 1(b) showed a decreasing pattern along with the formation of aerobic granules. SVI for the first 13 days of the formation process was noticeably higher compared to the rest of the formation days. However, biomass concentrations in terms of MLSS and MLVSS (Fig. 1(c)) were comparatively high between day 1 to 13 and day 41 to 77. These demonstrated that the transient phase in the formation process was strongly related to the settling properties of aerobic granules. Based on microscopic observation and SVI profile, it was more likely that aerobic granules on day 1 to 36 and day 41 to 67 were in aggregation and stability phases, respectively. During aggregation phase, formation of aerobic granules was highly dependable on OLR. This matched well with the profile of MLSS and MLVSS which varied according to changes in CODs influent from day 1 to 36. Cycle time was adjusted from 4 to 3 hours on day 33 when analysis on F/M ratio (Fig. S3) indicated that the biomass decreased due to insufficient food since 22 days of the formation process. Changes in CODs influent during the stability phase did not significantly affect MLSS and MLVSS profile. By removing the biofilm which has grown on the reactor wall during reactor maintenance on day 69 , aerobic granules did not have to outcompete for food with biofilm. In turn, MLSS promptly increased from $0.83 \mathrm{~g} \mathrm{~L}^{-1} \mathrm{on}$ day 67 to $1.38 \mathrm{~g} \mathrm{~L}^{-1}$ on day 71 .

Along with the increase in MLSS and partial disintegration of mature aerobic granules, it appeared that some aerobic granules floated and entrapped into effluent discharge pipe during feeding. As a result, MLSS promptly decreased from $1.58 \mathrm{~g} \mathrm{~L}^{-1}$ on day 77 to $0.60 \mathrm{~g} \mathrm{~L}^{-1}$ on day 83. It was expected that hydrolysis of anaerobic core during disintegration of mature aerobic granules releases gases and organics acids [24], which were entrapped within the sludge layer at static condition. Besides, long feeding time may create an anoxic condition inside the reactor and this governed the denitrification process which released nitrogen gas within the sludge layer. From observation, the aerobic granules started to float after 20 min of feeding time. Hence, the feeding time was changed from 30 to 10 min on day 82 with settling time remained at $15 \mathrm{~min}$ throughout the formation process. After that, a reduction in biomass concentration and sludge retention time (Fig. S3) was observed.

Nonetheless, mature aerobic granules were successfully re-dominated in the reactor on day 105 . The formation of these aerobic granules in airlift reactor with divided funnels was mostly stable between day 41 to 67 , and this was in line with result on $\mathrm{F} / \mathrm{M}$ ratio that varied slightly between 0.57 to $1.13 \mathrm{~g} \mathrm{CODs} \mathrm{g}^{-1} \mathrm{MLVSS}^{-1} \mathrm{~d}^{-1}$. Deng et al. [25] suggested a benchmark of F/M ratio above $0.5 \mathrm{~g} \mathrm{COD} \mathrm{g}^{-1} \mathrm{MLVSS}^{-1} \mathrm{~d}^{-1}$ to meet a normal growth, reproduction and a variety of physiological demand of the microbial. During stable periods, the differences of SVI in each 5 min intervals of settling time started to plateau off and were equivalently distributed. Aerobic granules were stabilized at average MLSS of $0.85 \mathrm{~g} \mathrm{~L}^{-1}$ and SRT of 6 days.

The enormous deviation in aerobic granules size obtained in this study was caused by self-aggregation of aerobic granules and re-granulation of the detached bioflocs, newborn flocs and crushed aged aerobic granules. The final aerobic granules size obtained on day 105 was in the range of 326 to $728 \mu \mathrm{m}$ and can be considered as smaller compared to that of reported by other studies [26]-[29] utilizing sewage as substrate which was around 750 to $800 \mu \mathrm{m}$, and can reach up to $2000 \mu \mathrm{m}$. This was mainly attributed to short flowing trajectories in the airlift reactor with divided funnels. The variations in OLR have intrusive the formation process particularly during low OLR, and required longer period to achieve mature aerobic granules or full granulation of biomass. Based on our previous study [9] on the formation of aerobic granules in SBR with equal H/D ratio, the use of divided funnels has successfully expedited the formation period of mature aerobic granules within 67 days (in this study) compared to 89 days (previous study). The result obtained enlightened that aerobic granule formation only required a definite critical height for an effective flowing trajectory to take place. Thus, this study supports the advantage of using an airlift reactor with divided funnels in optimizing the performance of reactor with low H/D ratio. 

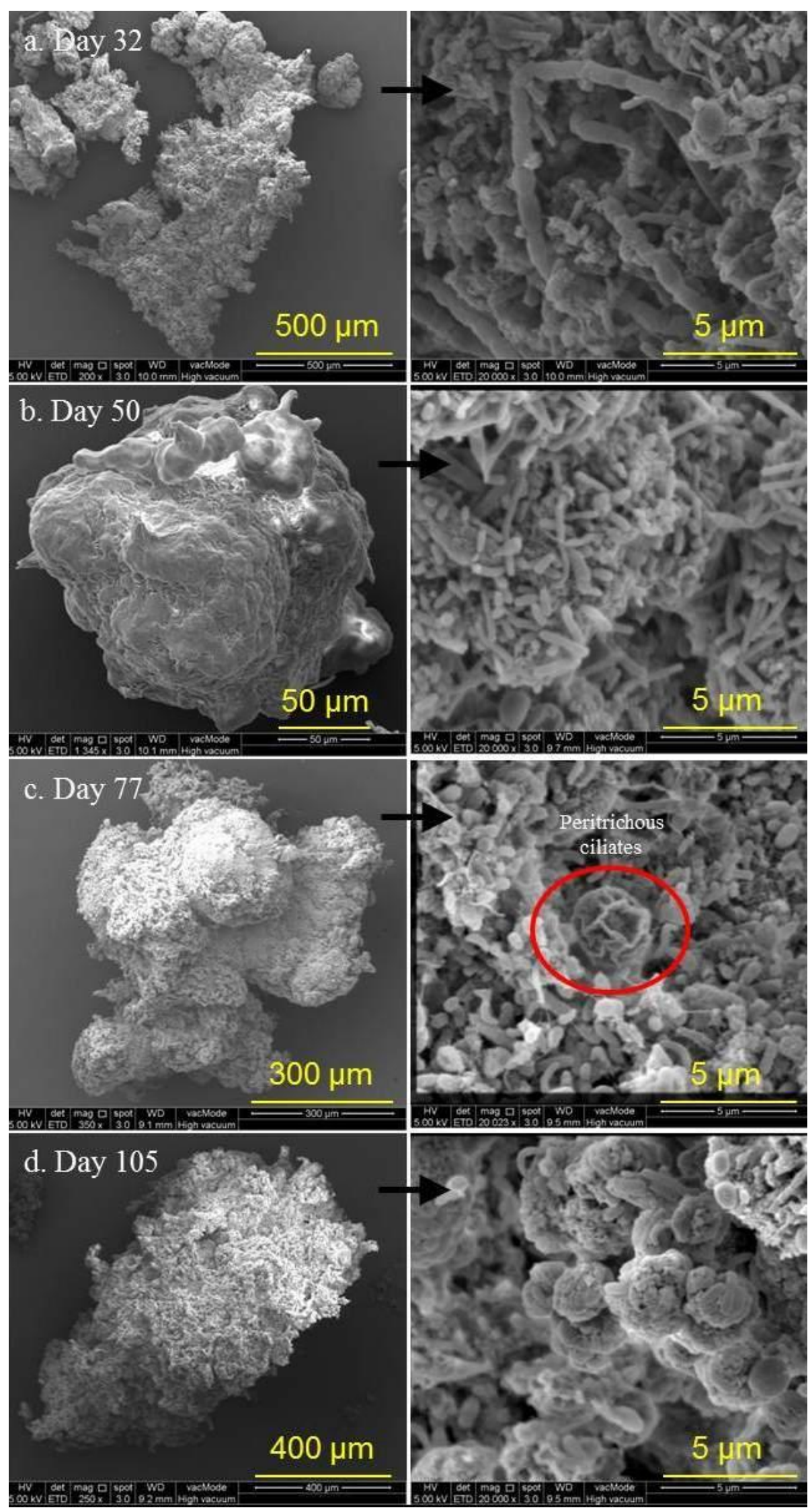

Fig. 2 - Morphological developments of aerobic granules during the experimental run. 


\subsection{Reactor Performance}

Fig. S4(a), Fig. S4(b) and Fig. S4(c) depict influent and effluent concentration as well as the removal efficiency of soluble CODs, SS and ammonium during the formation process, respectively. During the first 36 days, aerobic granules were in formation stage and thus led to poor CODs and SS removal. Negative removal for SS at an early stage indicated that the biomass was excessively washed out from the reactor. Afterwards, the reactor's performance improved continuously and was at stable condition throughout day 41 to 67, the average of CODs, SS and ammonium removal reached $71 \%, 64 \%$ and $78 \%$, respectively. As for the concentration of nitrite and nitrate in the effluent during stable periods, the respective average values of $14 \mathrm{mg} \mathrm{l}^{-1}$ and $13 \mathrm{mg} \mathrm{l}^{-1}$ were obtained.

The average of CODs, SS and ammonium removal obtained in this study can be considered as moderate compared to that of reported by other studies [26]-[29] utilizing sewage as substrate which was generally more than $90 \%$ and $80 \%$ for COD and ammonium, respectively. The moderate CODs removal and the decreasing patterns for $\mathrm{pH}$ at the end of reaction time (Fig. S2(d)) were attributed to low COD/ammonium ratios during Phase 1 and Phase 2, which was less than 6.0 (Fig. S2(d)). This finding can be associated to studies by Yang et al. [30] and Wang et al. [31] who postulated that aerobic granulation and nitrification process are closely related to the COD/nitrogen (COD/N) ratios applied to the reactors where inhibition of both heterotrophic and nitrifying bacteria activities may occur if substrate with COD/N ratio lower than 10 is used. On the other hand, Wagner and da Costa [32] reported a decrease in COD removal from $96 \%$ to $78 \%$ when COD/N ratio decreased from 11.2 to 4.7 , even though the influent ammonium concentration was less than $91 \mathrm{mg} \mathrm{l}^{-1}$. Thus, it can be concluded that the rate of nitrification inhibition should not be estimated solely based on ammonium influent concentration. Complete nitrification could occur even when the wastewater has high ammonium influent, and the COD/N ratio is over 10 [30].

\subsection{Distribution of EPS}

Fig. 3(a) shows the appearance of contrast image, while Fig. 3(b) to Fig. 3(e) display fluorescent staining results of the whole aerobic granules on day 77 and 105 . Fluorescent images were probed at the centre $(-72 \mu \mathrm{m})$ of aerobic granules for day 77, and $7 \mu \mathrm{m}$ from the centre of aerobic granules for day 105 (centre at $11 \mu \mathrm{m}$ ). The thicknesses of the whole aerobic granules used for CLSM image analysis on day 50, 77 and 105 were reflected on the length of Zsectioning as illustrated in Fig. 3(f). The condition of selected aerobic granules for CLSM image viewing for day 77 was matured aerobic granules with apparent existence of filamentous microorganism protruding from aggregates. Whilst, selected aerobic granules for CLSM image viewing for day 105 were from partial disintegration of aerobic granules on day 91.

Based on the image analysis at different Z-sectioning on day 77, it can be concluded that the core of aerobic granules was formed by protein, whereas the active cells (nucleic acid) and $\beta$-D-glucopyranose polysaccharides were also within the core of the granule, but to a lesser extent than protein. This finding was consistent with reports by Chen et al. [21] and McSwain et al. [33]. Chen et al. [21] reported that $\beta$-D-glucopyranose polysaccharides are concentrated not only in the outer layer, but they also spread throughout the granules interior. Meanwhile, McSwain et al. [33] spotted few signals of active cells from aerobic granule centre and claimed that the sample was either in flocs or small aerobic granules formed when cells and EPS existed together at the centre.

Zhu et al. [34] further described a study by Wang et al. [35] on $\beta$ - polysaccharides forming a continuous shell and integrated structure that held the soft core of aerobic granules as mesh skeleton which favoured the formation of stable granular structure. This was in conjunction with the overlapped images on day 77 which revealed that $\beta$-Dglucopyranose polysaccharides were likely to entrench the protein surface with traces of active cell almost completely covered. Whilst, the overlapped images on day 105 signified that $\beta$-D-glucopyranose polysaccharides were likely to act as the backbone for protein and active cells layer embedment. This observation was supported by Adav et al. [36], in their study revealing large fragments which may have originated from the outer layers of aerobic granules prior to hydrolysis. The $\beta$-polysaccharides were likely to form the backbone of a network like outer layer of embedded proteins, lipids, $\alpha$-polysaccharides and cells, whose elasticity supported the mechanical stability of granule.

Image analysis at different Z-sectioning on day 50 (image not included) was also similar to day 77. Only protein, with a few spots of $\beta$-D-glucopyranose polysaccharides, was present on the overlapped images of day 50. In contrast, the overlapped image on day 105 which was probed at $7 \mu \mathrm{m}$ from the centre of aerobic granules indicated the significant presence of active cells. Furthermore, the image analysis at different Z-sectioning on day 50, 77 and 105 showed that high fluorescent intensity for active cells was present between $\beta$-D-glucopyranose polysaccharides and protein in a very thin layer depending on the thickness and form of aerobic granules. Outer surface and core of aerobic granules were mainly composed of $\beta$-D-glucopyranose polysaccharides and protein, respectively.

\subsection{Microbial Community During Granulation}

In order to demonstrate the evolution of microbial population, a total of 58,679 effective sequencing readings were assigned according to Bergey's taxonomy. Analysis was carried out on day 0, 8, 50, 77 and 105, each representing seed, aggregation, stability, disintegration and re-aggregation phase, respectively. Most of the effective sequencing 
readings were affiliated to 3 major phyla out of 25 detected phyla in total, which were Proteobacteria, Bacteroidetes and Firmicutes. Previous studies reported that phyla of Bacteroidetes [37], Proteobacteria [25] and Flavobacterium [38] were dominant in aerobic granules formation.

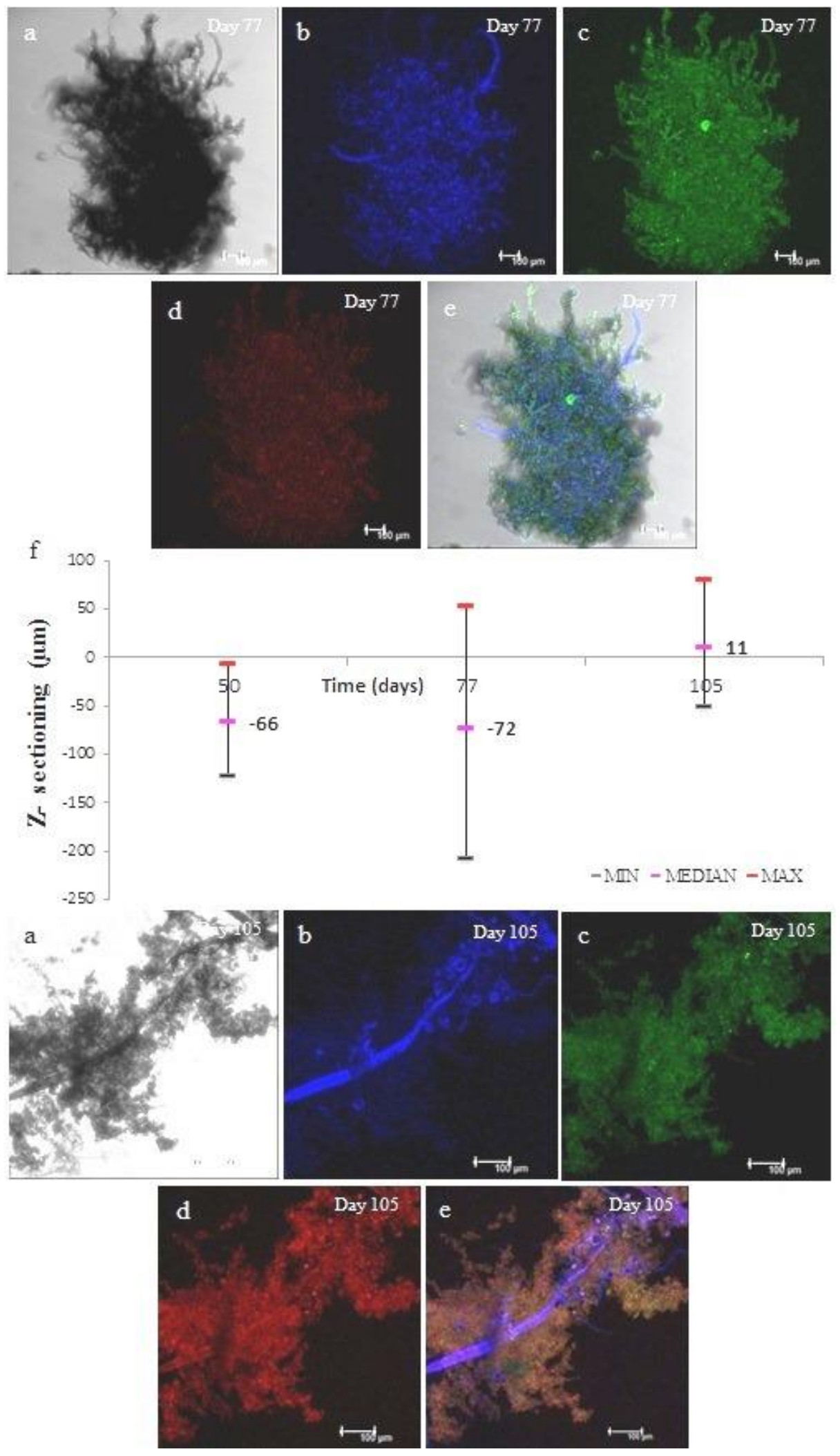

Fig. 3 - CLSM images of aerobic granules on day 77 and 105; (a) Contrast images, (b) $\beta$-D-glucopyranose polysaccharides (calcofluor white), (c) Protein (FITC), (d) Nuclei acid (SYTO 63), (e) Overlap images of b-d and (f) Z-sectioning on whole aerobic granules on day 50, 77 and 105. 
Cluster analysis as shown in Fig. 4 was used to scrutinize the similarity of microbial community that represented different phases in aerobic granules formation. It was noted that Betaproteobacteria and Sphingobacteriia classes, which belong to the Proteobacteria and Bacteroidetes phyla, were always dominant throughout the formation period. The rapid increase in total sequences percentage for Betaproteobacteria and Sphingobacteriia classes from $42 \%$ in seed to $74 \%, 68 \%$ and $62 \%$ on day 50,77 and 105, respectively, indicated a significant shift in microbial population. Dominant classes have outcompeted minor classes in which $18 \%$ of Bacilli classes was markedly existed only on day 8 , suggesting that this class was beneficial to the aggregation process at an early stage. Bacteroidia and Nitrospira classes existed noticeably in seed, with Flavobacteriia class increased steadily throughout the formation period. This demonstrated that only certain microbial community was beneficial to aerobic granules formation.

similarity

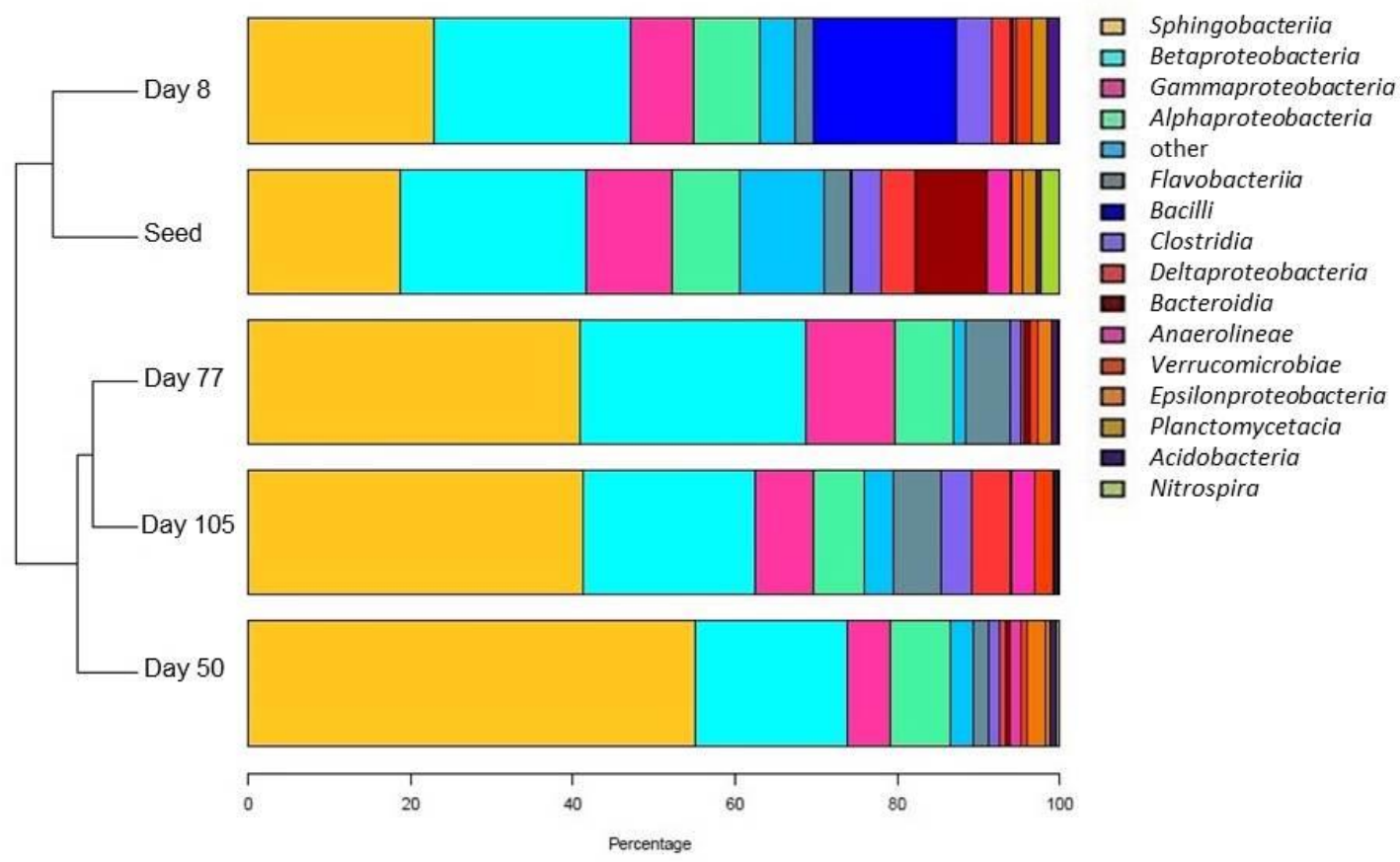

Fig. 4 - Cluster analyses for the first 15 taxonomic classifications from bacterial community of seed, day 8, 50, 77 and 105 at the class level

As shown in Fig. 5, the taxonomic classification of pyrosequences from bacterial community at genus level revealed that seed contained a greater microbial diversity than aerobic granules. For example, the presence of Candidatus, vadinBC27, unclassified_Rhodocyclaceae and Sulfuritale genera in seed were over 3\% as compared to $1 \%$ in aerobic granules which was formed afterwards. Except for several dominant genus, most of the microbial community from seed was suppressed to less than $1 \%$ during the aggregation, stability, disintegration and re-aggregation phases. Bacillus genus was markedly present in aggregation phase (day 8) but almost non-existent in the late formation process, suggesting that this genus was dominant in smaller aerobic granules with median size of $99 \mu \mathrm{m}$ and eventually was eliminated as the aerobic granules size increased.

Genus of Thauera from Rhodocyclaceae family also was significantly prevalent on day 8 with nearly $15 \%$ abundance, but continued to decrease to less than $5 \%$ on day 50 when the median aerobic granules size was $336 \mu$ m. Then, Comamonas, Alicycliphilus and Ottowia genus from Comamonadaceae family increased with the decrease in Rhodocyclaceae class on day 50, 77 and 105 with total abundance accounted at 8\%, 18\% and 10\%, respectively. Thus, this highlighted the shift in microbial community for the denitrification process from Rhodocyclaceae to Comamonadaceae family which was accommodated with changes in aerobic granules size from $147 \mu \mathrm{m}$ to more than $336 \mu \mathrm{m}$. This result has confirmed an increasing trend in the dominant abundance of unclassified_Chitinophagaceae and unclassified_Saprospiraceae from Sphingobacteriia class throughout the formation period. Further investigation on the phylogenetic analysis using MEGAN software showed a shift in Chitinophagaceae family, from Ferruginibacter genus in seed, to Sediminibacterium genus on day 8 and Taibaiella genus on day 50 and 77, and Filimonas genus on day 105. The closed up morphology image for aerobic granules on day 105 (Fig. 2(d)) proved the domination of unclassified_Chitinophagaceae genus, where rods cells with rounded or so called microcysts were tightly linked together to form a compact flora structure. 

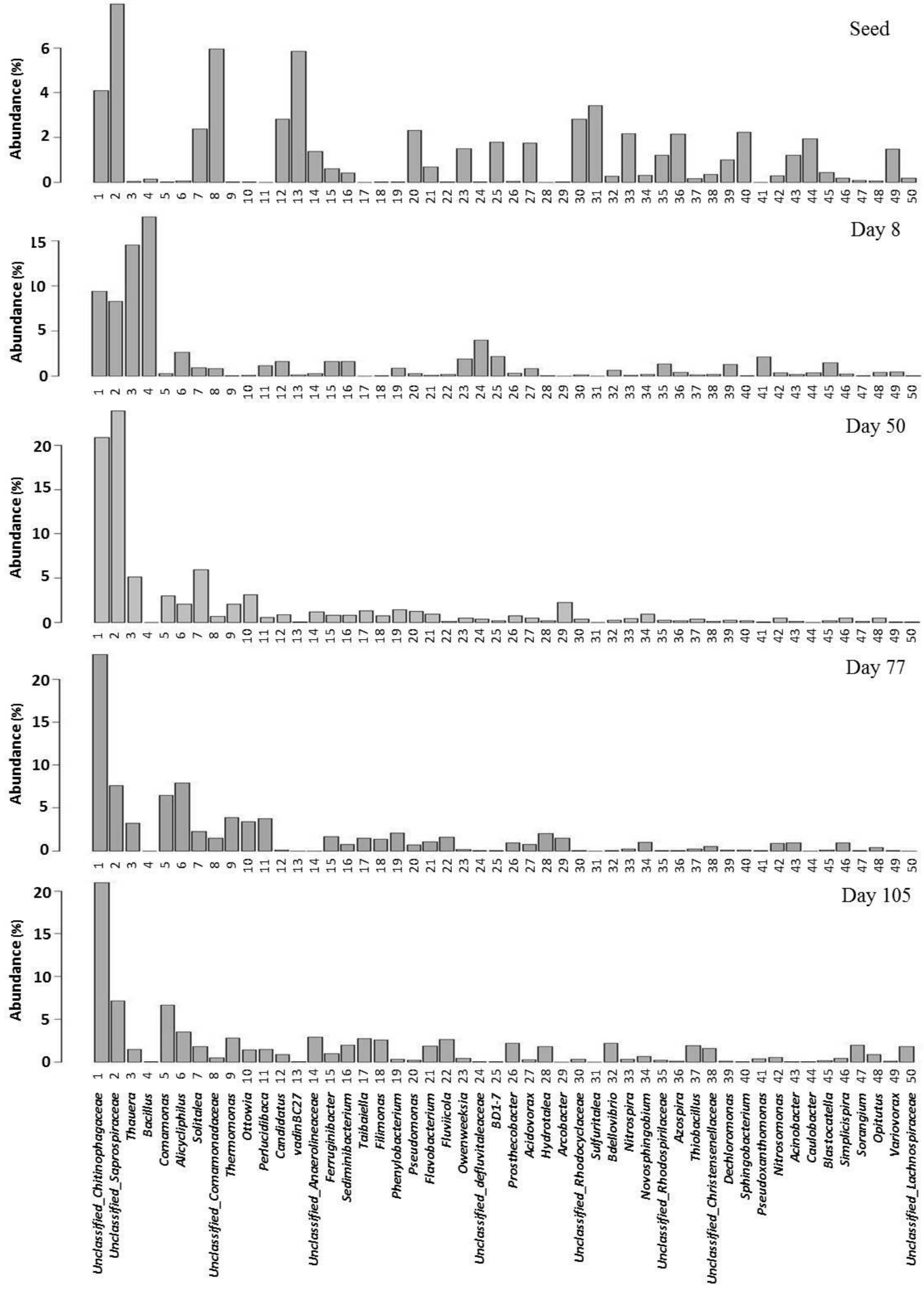

Fig. 5 - Taxonomic classification of pyrosequences from bacterial community of seed, day $8,50,77$ and 105 at the genus level 
Comparing the result of microbial community in this study with other research works further proves that the dominance of a particular microbial is governed by substrate or type of wastewater and OLR. The dominant microbial in seed will suppress the existence of other species over the formation period and results in low microbial species diversity in mature aerobic granules. A study by Li et al. [38] confirmed that reactor with high OLR has the lowest microbial species diversity compared to reactor with low OLR. Zhou et al. [39] proved that microbial selection pressure is not a prerequisite for aerobic granulation from both the dynamic granulation steps and molecular biology aspects. For example, the domination of Sphingobacteriia class in this study was similar to that of reported by Zhou et al. [39] on the formation of aerobic granules in sewage. Findings by Xia et al. [40] suggested a gradual accumulation of antibioticresistant microorganisms like Firmicutes sp. in the treatment of sewage containing antibiotics. Whilst, Abdullah et al. [37] claimed that the existence of Propionicbacteriaceae family is associated with the presence of phenolic compounds.

\section{Summary}

Aerobic granules were successfully attained although the combined selective pressure applied was not favourable for aerobic granules formation. Although the variations in OLR have intrusive the formation of AGS particularly during low OLR, the self-aggregation of AGS and re-granulation of the detached bioflocs, newborn flocs and crushed aged AGS ensured the later stability of AGS system. Smaller size of aerobic granules in the range of 333 to $750 \mu \mathrm{m}$ was relatively stable. This was based on CLSM analysis that showed active cells, $\beta$-D-glucopyranose polysaccharides and protein existed together at aerobic granules core. The NGS analysis result indicated the shift in microbial community performing denitrification process from Rhodocyclaceae to Comamonadaceae class was accommodated with the changes in aerobic granules size from $147 \mu \mathrm{m}$ to more than $336 \mu \mathrm{m}$.

\section{Acknowledgments}

This work was financially supported by the Department of Higher Education Malaysia (FP041-2013B) and University of Malaya research grants (RG160-12SUS and PV038-2012A). Awang N. A acknowledges the Ministry of Education Malaysia and Universiti Sains Malaysia for their support in her PhD fellowship.

\section{References}

[1] Lee, D. J., Chen, Y. Y., Show, K. Y., Whiteley, C. G. and Tay, J. H. (2010). Advances in aerobic granule formation and granule stability in the course of storage and reactor operation. Biotechnology Advances, 28(6), 919-934.

[2] Liu, X. W., Sheng, G. P. and Yu, H. Q. (2009). Physicochemical characteristics of microbial granules. Biotechnology Advances, 27(6), 1061-1070.

[3] Zhu, L., Lv, M., Dai, X., Zhou, J. and Xu, X. (2013). The stability of aerobic granular sludge under 4-chloroaniline shock in a sequential air-lift bioreactor (SABR). Bioresource Technology, 140, 126-130.

[4] Long, B., Yang, C. Z., Pu, W. H., Yang, J. K., Jiang, G. S., Li, C. Y., Liu, F. B., Dan, J. F., Zhang, J. and Zhang, L. (2016). Rapid cultivation of aerobic granule for the treatment of solvent recovery raffinate in a bench scale sequencing batch reactor. Separation and Purification Technology, 160, 1-10.

[5] Sheng, G. P., Yu, H. Q. and Li, X. Y. (2010). Extracellular polymeric substances (EPS) of microbial aggregates in biological wastewater treatment systems: a review. Biotechnology Advances, 28(6), 882-894.

[6] Chen, Y., Jiang, W., Liang, D. T. and Tay, J. H. (2007). Structure and stability of aerobic granules cultivated under different shear force in sequencing batch reactors. Applied Microbiology and Biotechnology, 76(5), 1199-1208.

[7] Tao, J., Qin, L., Liu, X., Li, B., Chen, J., You, J., Shen, Y. and Chen,BX. (2017) Effect of granular activated carbon on the aerobic granulation of sludge and its mechanism, Bioresource Technology, 236, 60-67.

[8] Verawaty, M., Tait, S., Pijuan, M., Yuan, Z. and Bond, P. L. (2013). Breakage and growth towards a stable aerobic granule size during the treatment of wastewater. Water Research, 47(14), 5338-5349

[9] Awang, N. A. and Shaaban, M. G. (2016) Effect of reactor height/diameter ratio and organic loading rate on formation of aerobic granular sludge in sewage treatment, International Biodeterioration \& Biodegradation, 112, $1-11$.

[10] Val Del Río, A., Figueroa, M., Mosquera-Corral, A., Campos, J. L. and Méndez, R. (2013) Stability of aerobic granular biomass treating the effluent from a seafood industry, International Journal of Environmental Research, 7, 265-276.

[11] Corsino, S. F., di Biase, A., Devlin,T. R., Munz, G., Torregrossa, M. and Oleszkiewicz, J. A (2017) Effect of extended famine conditions on aerobic granular sludge stability in the treatment of brewery wastewater, Bioresource Technology, 226, 150-157.

[12] Liu, Y. Q. and Tay, J. H. (2012). The competition between flocculent sludge and aerobic granules during the longterm operation period of granular sludge sequencing batch reactor. Environmental Technology, 33(23), 2619-2626. 
[13] Pronk, M., de Kreuk, M. K., de Bruin, B., Kamminga, P., Kleerebezem, R. and van Loosdrecht, M. C. M. (2015). Full scale performance of the aerobic granular sludge process for sewage treatment. Water Research, 84, $207-217$.

[14] Nancharaiah, Y. V. and Kiran Kumar Reddy, G. (2018) Aerobic granular sludge technology: Mechanisms of granulation and biotechnological applications. Bioresource Technology, 247, 1128-1143.

[15] Awang, N. A. and Shaaban, M. G. (2015). Impact of height to diameter (H/D) ratio on aerobic granular sludge (AGS) formation in sewage treatment. Jurnal Teknologi (Sciences \& Engineering), 77:32, 95-103.

[16] Liu, Y. and Tay, J. H. (2004). State of the art of biogranulation technology for wastewater treatment. Biotechnology Advances, 22(7), 533-563.

[17] Liu, Y. Q. and Tay, J. H. (2007). Cultivation of aerobic granules in a bubble column and an airlift reactor with divided draft tubes at low aeration rate. Biochemical Engineering Journal, 34(1), 1-7.

[18] Liu, Y. and Tay, J. H. (2002). The essential role of hydrodynamic shear force in the formation of biofilm and granular sludge. Water Research, 36(7), 1653-1665.

[19]Zhoua, J. H, Zhang, Z. M., Zhao, H., Yu, H. T., Alvarez, P. J. J., Xua, X. Y. and Zhua, L. (2016) Optimizing granules size distribution for aerobic granular sludge stability: Effect of a novel funnel-shaped internals on hydraulic shear stress, Bioresource Technology, 216, 562-570.

[20] APHA. (2005) Standard Methods for the Examination of Water and Wastewater, $21^{\text {st }}$ ed., American Public Health Association/American Water Works Association/Water Environment Federation, Washington, DC, USA.

[21] Chen, M. Y., Lee, D. J., Tay, J. H. and Show, K. Y. (2007). Staining of extracellular polymeric substances and cells in bioaggregates. Applied Microbiology and Biotechnology, 75(2), 467-474.

[22] Liu, Y., Yang, S. F. and Tay, J. H. (2004). Improved stability of aerobic granules by selecting slow-growing nitrifying bacteria. Journal of Biotechnology, 108(2), 161-169.

[23] Weber, S. D., Ludwig, W., Schleifer, K. H. and Fried, J. (2007). Microbial composition and structure of aerobic granular sewage biofilms. Applied and Environmental Microbiology, 73(19), 6233-6240.

[24] Tay, J. H., Ivanov, V., Pan, S. and Tay, S. T. L. (2002). Specific layers in aerobically grown microbial granules. Letters in Applied Microbiology, 34(4), 254-257.

[25] Deng, S., Wang, L. and Su, H. (2016). Role and influence of extracellular polymeric substances on the preparation of aerobic granular sludge, Journal of Environmental Management, 173, 49-54.

[26] Ni, B. J., Xie, W. M., Liu, S. G., Yu, H. Q., Wang, Y. Z., Wang, G. and Dai, X. L. (2009). Granulation of activated sludge in a pilot-scale sequencing batch reactor for the treatment of low-strength municipal wastewater. Water Research, 43(3), 751-761.

[27] Peyong, Y. N., Zhou, Y., Abdullah, A. Z. and Vadivelu, V. (2012). The effect of organic loading rates and nitrogenous compounds on the aerobic granules developed using low strength wastewater. Biochemical Engineering Journal, 67, 52-59.

[28]Liu, Y. Q., Moy, B. Y. P. and Tay, J. H. (2007). COD removal and nitrification of low-strength domestic wastewater in aerobic granular sludge sequencing batch reactors. Enzyme and Microbial Technology, 42(1), 23-28.

[29] Su, B., Cui, X. and Zhu, J. (2012). Optimal cultivation and characteristics of aerobic granules with typical domestic sewage in an alternating anaerobic/aerobic sequencing batch reactor. Bioresource Technology, 110, 125129.

[30] Yang, S. F., Tay, J. H. and Liu, Y. (2004). Inhibition of free ammonia to the formation of aerobic granules. Biochemical Engineering Journal, 17(1), 41-48.

[31] Wang, X. H., Zhang, H. M., Yang, F. L., Xia, L. P. and Gao, M. M. (2007). Improved stability and performance of aerobic granules under stepwise increased selection pressure. Enzyme and Microbial Technology, 41(3), $205-211$.

[32] Wagner, J. and da Costa, R. H. R. (2013). Aerobic Granulation in a Sequencing Batch Reactor Using Real Domestic Wastewater. Journal of Environmental Engineering, 139(11), 1391-1396.

[33] Mcswain, B. S., Irvine, R. L., Hausner, M. and Wilderer, P. A. (2005). Composition and Distribution of Extracellular Polymeric Substances in Aerobic Flocs and Granular Sludge. Applied and Environmental Microbiology, 71(2), 1051-1057.

[34]Zhu, L., Lv, M., Dai, X., Yu, Y., Qi, H. and Xu, X. (2012). Role and significance of extracellular polymeric substances on the property of aerobic granule. Bioresource Technology, 107, 46-54.

[35] Wang, Z. W., Liu, Y. and Tay, J. H. (2005). Distribution of EPS and cell surface hydrophobicity in aerobic granules. Applied Microbiology and Biotechnology, 69(4), 469-473.

[36] Adav, S. S., Lee, D. J., \& Tay, J. H. (2008). Extracellular polymeric substances and structural stability of aerobic granule. Water Research, 42(6-7), 1644-1650.

[37] Abdullah, N., Yuzir, A., Curtis, T. P., Yahya, A. and Ujang, Z. (2013). Characterization of aerobic granular sludge treating high strength agro-based wastewater at different volumetric loadings. Bioresource Technology, 127, 181187.

[38] Li, A., Yang, S., Li, X. and Gu, J. (2008b). Microbial population dynamics during aerobic sludge granulation at different organic loading rates. Water Research, 42(13), 3552-3560. 
[39]Zhou, D., Niu, S., Xiong, Y., Yang, Y. and Dong, S. (2014). Microbial selection pressure is not a prerequisite for granulation: dynamic granulation and microbial community study in a complete mixing bioreactor. Bioresource Technology, 161, 102-108.

[40] Xia, Z., Xiao-Chun, W., Zhong-Lin, C., Hao, X., and Qing-Fang, Z. (2015). Microbial community structure and pharmaceuticals and personal care products removal in a membrane bioreactor seeded with aerobic granular sludge. Applied Microbiology and Biotechnology, 99, 425-433. 